\title{
SOME REMARKS ON LOCALLY NILPOTENT GROUPS OF FINITE RANK
}

\author{
HOWARD SMITH ${ }^{1}$
}

\begin{abstract}
The main subclasses of locally nilpotent groups are considered in conjunction with the extra hypothesis of finite rank or an allied property. These classes are ordered by inclusion, examples being indicated wherever inclusion is proper.
\end{abstract}

There are several properties of nilpotent groups each of which, if possessed by a group $G$, is sufficient to ensure that $G$ is locally nilpotent. For instance (in ascending order of generality), there are the classes of nilpotent, Fitting, Baer and Gruenberg groups. Also to be found among the Gruenberg groups are those satisfying the normalizer condition, which in turn include both the hypercentral groups and groups in which all subgroups are subnormal. All these classes of groups are known to be distinct, and the only unsettled question with regard to class inclusion is whether a group with all subgroups subnormal need be a Fitting group (see Chapter $6, \S \S 1$ and 3 of [2] for a summary of these matters and for a statement and proof of the theorem of $\mathrm{Mal}^{\prime} \mathrm{cev}$ mentioned below). The purpose of this note is to show that, if attention is restricted to groups having finite rank, or the more general property of finite abelian subgroup rank (a class denoted here by $\left.\mathfrak{F}_{r(\mathrm{ab})}\right)$, then the hierarchy for the above classes can be completely determined.

From results of $\mathrm{Mal}^{\prime} \mathrm{cev}$ we know that the locally nilpotent group $G$ belongs to $\mathfrak{F}_{r(\mathrm{ab})}$ if and only if the torsion subgroup of $G$ is a direct product of Cernikov $p$ groups and its factor group in $G$ is nilpotent of finite rank. Since such a group is also hypercentral, three of our properties coincide with local nilpotency for groups in $\mathfrak{F}_{r(\mathrm{ab})}$ and thus for groups with finite rank also. The example given in Theorem 1 of $[\mathbf{4}]$ is of a nonnilpotent group of rank 2 in which all subgroups are subnormal, while in [5] it is shown that a Baer group of finite rank is a Fitting group, although such a group need not of course have all its subgroups subnormal (see, for instance, the example given at the end of $\S 2$ of $[6]$ ). The wreath product of a group of type $C_{p^{\infty}}$ by a group of order $p$, a prime, is not a Baer group, but is locally nilpotent and has finite rank. The classification referred to above will thus be completed by proving the following two results. (The second of these states far more than is required but is possibly of independent interest.)

THEOREM 1. A group in the class $\mathfrak{F}_{r(\mathrm{ab})}$ in which every subgroup is subnormal is a Fitting group.

THEOREM 2. There is a group in $\mathfrak{F}_{r(\mathrm{ab})}$ which is generated by two subnormal abelian subgroups but which is not a Fitting group.

Received by the editors March 23, 1983 and, in revised form, December 20, 1983.

1980 Mathematics Subject. Classification. Primary $20 \mathrm{~F} 19$.

${ }^{1}$ The author is grateful for assistance received from the Department of Mathematics at York University. 
Now a periodic group which satisfies the hypotheses of Theorem 1 is in fact nilpotent [4, Lemma 4], and so the first result follows from

LEMMA 3. Let $G$ be a Baer group, and suppose $N$ is a normal nilpotent subgroup of $G$ such that $G / N$ has finite rank. Then $G$ is a Fitting group.

ProOf. Suppose $N$ has nilpotency class $c$ and that $G / N$ has rank $r$, where $G$ is a Baer group. Assume $\langle x\rangle \triangleleft^{m} G$, for some arbitrary element $x$ of $G$. We must show that $\langle x\rangle^{G}$ is nilpotent. From [5], we have that $\langle x\rangle^{G}$ is nilpotent modulo $N$. Let $K=\langle x\rangle^{H}$, where $H$ is an arbitrary finitely generated subgroup of $G$, and write $M=K \cap N$. Then it suffices to prove that, for a suitably bounded integer $d,\left[M,{ }_{d} K\right]=1$. Since the intersection of all characteristic subgroups of prime-power index in $M$ is trivial, we may assume that $M$ is a finite $p$-group, for some prime $p$. For each $i=1, \ldots, c$, write $\bar{\gamma}_{i}=\gamma_{i}(M) / \gamma_{i+1}(M), C_{i}=C_{K}\left(\bar{\gamma}_{i}\right)$. Then $K_{i}=K / C_{i}$ is also a finite $p$-group. Further, $M \leq C_{i}$ and so $K_{i}$ has rank at most $r$. Let $\left.D_{i}=\bar{\gamma}_{i}\right] K_{i}$. By Burnside's Basis Theorem, there are elements $h_{1}, \ldots, h_{r}$ of $H$ such that $K_{i}=\left\langle x^{h_{j}} C_{i}, j=1, \ldots, r\right\rangle$. Since $\left\langle x^{h_{j}}\right\rangle \triangleleft^{m-1} K$, for each $j$, it follows that $\left[D_{i, m}\left\langle x^{h_{j}}\right\rangle C_{i}\right] \leq \bar{\gamma}_{i}$ and hence that $\left[D_{i, 2 m}\left\langle x^{h_{j}}\right\rangle C_{i}\right]=1$. Then, using Lemma 5 of $[\mathbf{1}]$ and a simple inductive argument, we deduce that the subnormal defect of $K_{i}$ in $D_{i}$ is at most $d_{i}=d_{i}(2 m, r)$. Thus $\left[\bar{\gamma}_{i}, d_{i} K_{i}\right]=1$, that is, $\left[\gamma_{i}(M), d_{i} K\right] \leq \gamma_{i+1}(M)$. Writing $d=d_{1}+d_{2}+\cdots+d_{c}$, we see that $\left[M,{ }_{d} K\right]=1$, as required.

Proof of Theorem 2. Suppose that, for each odd prime $p$, we have constructed a finite $p$-group $G_{p}$ with the following properties.

(i) $G_{p}$ is generated by a cyclic subgroup $\left\langle\alpha_{p}\right\rangle$ and an abelian subgroup $H_{p}$.

(ii) $\left\langle\alpha_{p}\right\rangle \triangleleft^{u} G_{p}, H_{p} \triangleleft^{v} G_{p}$, where $u, v$ do not depend on $p$.

(iii) The nilpotency class of $\left[\left\langle\alpha_{p}\right\rangle, G_{p}\right]$ is at least $n(p)$, where $p<q \Rightarrow n(p)<n(q)$ (all we require is arbitrarily large class).

Then the direct product $G$ of the subgroups $G_{p}$ is clearly a Baer group of finite abelian subgroup rank. To complete the construction, we introduce an element $\beta$, of infinite order, and define an action of $\langle\beta\rangle$ on $G$ as follows: for each $x$ in $G_{p}$, and for each prime $p, x^{\beta}=x^{\alpha_{p}}, x^{\beta^{-1}}=x^{\alpha_{p}^{-1}}$. Let $\left.G^{*}=G\right]\langle\beta\rangle$. Then $\left[\langle\beta\rangle, G^{*}\right]$, and hence $\langle\beta\rangle^{G^{*}}$, is not nilpotent, although $G^{*}$ is a Baer group (of torsion-free rank 1), generated by subnormal abelian subgroups $H=D_{p}^{r} H_{p}$ and $K=\left\langle\beta, \alpha_{p}\right.$, for all $\left.p\right\rangle$.

Now let $p$ be a prime, $n$ a given integer. To obtain our group $G_{p}$ we follow, with appropriate modifications, a construction due to Roseblade and Stonehewer [3, Theorem E]. (It should perhaps be remarked that the construction which follows can be described in purely group-theoretic terms in a manner similar to that employed in the proof of $[6$, Theorem 5.1], but the necessary computations become rather tedious.)

Let $F$ be a field of $p$ elements and suppose $A$ is an $n$-dimensional vector space over $F$ with basis $\left\{a_{1}, a_{2}, \ldots, a_{n}\right\}$. Let $T$ be the tensor algebra on $A$ (that is, $T=F+\sum_{j \geq 1} A_{j}$, where $A_{j}$ denotes the tensor product of $j$ copies of $A$, and multiplication is such that, if $x \in A_{j}$ and $y \in A_{k}$, then $x y \in A_{j+k}$ etc.), and write $R=T / I$, where $I$ is the ideal of $T$ generated by $\left\{a^{2}: a \in A\right\}$. We define $2 \times 2$ 
matrices over $R$ as follows:

$$
\alpha=\left(\begin{array}{ll}
1 & 0 \\
1 & 1
\end{array}\right), \quad \lambda(a)=\left(\begin{array}{ll}
1 & a \\
0 & 1
\end{array}\right), \quad \text { for each } a \in A .
$$

Set $K=\langle\alpha\rangle, L=\langle\lambda(a): a \in A\rangle, J=\langle L, K\rangle$. Then $L^{J}$ and $K^{J}$ may each be shown to be nilpotent of class 2 (see [3, Lemma 6]).

Write $V_{1}=\{(x, 0): x \in R\}, V_{2}=\{(0, x): x \in R\}$ and let $V$ be the direct sum of $V_{1}$ and $V_{2}$. Then let $H=L V_{2}$ in the natural split extension $G_{p}$ of $V$ by $J$. Since $[V, K, K]=1$ and $[V, L] \leq V_{2}$, we see that $K$ and $H$ are subnormal of defect at most 5,4 respectively in $G_{p}$. We show that $\left[K, G_{p}\right]$ has nilpotency class at least $n$. For each $i=1, \ldots, n$, let

$$
\delta\left(a_{i}\right)=\left[\alpha, \lambda\left(a_{i}\right)\right]=\left(\begin{array}{cc}
1-a_{i} & 0 \\
a_{i} & 1+a_{i}
\end{array}\right) .
$$

Then $[(0,1), \alpha]=(1,0)$ is contained in $\left[K, G_{p}\right]$, and so $\left[(1,0), \delta\left(a_{1}\right), \ldots, \delta\left(a_{n-1}\right)\right]=$ $\left((-1)^{n-1} a_{1} \cdots a_{n-1}, 0\right)$ is a nontrivial commutator of length $n$ in $\left[K, G_{p}\right]$, thus completing the proof.

\section{REFERENCES}

1. J. C. Lennox and S. E. Stonehewer, The join of two subnormal subgroups, J. London Math. Soc. (2) 22 (1980), 460-466.

2. D. J. S. Robinson, Finiteness conditions and generalised soluble groups, Vol. 2, Springer, Berlin, Heidelberg and New York, 1972.

3. J. E. Roseblade and S. E. Stonehewer, Subjunctive and locally coalescent classes of groups, J. Algebra 8 (1968), 423-435.

4. H. Smith, Hypercentral groups with all subgroups subnormal, Bull. London Math. Soc. 15 (1983), 229-234.

5. __ A note on Baer groups of finite rank, Math. Z. 184 (1983), 139-140.

6. __ Groups with the subnormal join property (to appear).

Department of Mathematics, York University, 4700 Keele Street, DownsVIEW, ONTARIO, CANADA 